\title{
Generating finite element method in constructing complex-shaped multigrid finite elements
}

\author{
Aleksandr Matveev ${ }^{1, *}$ \\ ${ }^{1}$ ICM SB RAS, 50, bil. 44, Akademgorodok, Krasnoyarsk, Russia, 660036
}

\begin{abstract}
The calculations of three-dimensional composite bodies based on the finite element method with allowance for their structure and complex shape come down to constructing high-dimension discrete models. The dimension of discrete models can be effectively reduced by means of multigrid finite elements (MgFE). This paper proposes a generating finite element method for constructing two types of threedimensional complex-shaped composite $\mathrm{MgFE}$, which can be briefly described as follows. An MgFE domain of the first type is obtained by rotating a specified complex-shaped plane generating single-grid finite element (FE) around a specified axis at a given angle, and an $\mathrm{MgFE}$ domain of the second type is obtained by the parallel displacement of a generating FE in a specified direction at a given distance. This method allows designing $\mathrm{MgFE}$ with one characteristic dimension significantly larger (smaller) than the other two. The MgFE of the first type are applied to calculate composite shells of revolution and complex-shaped rings, and the $\mathrm{MgFE}$ of the second type are used to calculate composite cylindrical shells, complex-shaped plates and beams. The proposed MgFE are advantageous because they account for the inhomogeneous structure and complex shape of bodies and generate low-dimension discrete models and solutions with a small error.
\end{abstract}

\section{Introduction}

The finite element method (FEM) [1, 2] is widely used to study the stress-strain state (SSS) of complex-shaped elastic composite bodies. In order to simplify calculations, the deformation of bodies of a certain type (for example, beams, plates, shells) is described using engineering theories based on hypotheses [3-9]. However, engineering solutions do not always meet modern requirements. Calculating composite bodies on the basis of the FEM in the formulation of a threedimensional problem of the elasticity theory [10] with account for their structure can be reduced to constructing high-dimension discrete models, of the order of $10^{9} \div 10^{12}$. For such discrete models, it is difficult to use computational software such as ANSYS, NASTRAN, etc. [2]. The dimension of discrete models can be effectively reduced by means of the MgFE [11-18], which also serve as a basis for the multigrid finite element method (MFEM) [13-17], based on the FEM

\footnotetext{
*Corresponding author: mtv241@mail.ru
} 
algorithms. The main advantages and features distinguishing the MFEM from the FEM are as follows.

1. In the MFEM (without increasing the dimension of MgFE), one can use arbitrarily small basic partitions of bodies, i.e., MgFE, which allows one to arbitrarily accurately account for their complex shape and inhomogeneous structure, as well as the complex nature of fixing and loading bodies. In the FEM, it is impossible to use arbitrarily small basic partitions because the PC resources are limited, which means that the MFEM is more effective than the FEM.

2. Application the MFEM (based on the basic models of bodies) requires a significantly smaller amount of RAM $\left(10^{3} \div 10^{6}\right.$ times) and time compared to the FEM used for basic models, i.e., the MFEM is more economical than FEM.

3. The MFEM uses homogeneous and composite MgFE constructed using nested grids, which expands the field of application of this method. The FEM uses single-grid homogeneous finite elements (FE). It is noteworthy that boundary-value problems can always be solved using the MFEM instead of the FEM because MgFE can always be used instead of single-grid FE. As $m \mathrm{gFE}$ are constructed using $m$ nested grids instead of one $(m>1)$, the MFEM can be regarded as the generalization of the FEM, i.e., the FEM is a special case of the MFEM. Hence, if the FEM-based calculations of bodies are carried out using MgFE, then this case is essentially the implementation of the MFEM.

In this paper, the two types of complex-shaped three-dimensional composite MgFE are designed using the generating FEM. According to this method, the MgFE domain is obtained using the specified displacement of a given complex-shaped plane single-grid FE (below referred to as a generating FE) in a three-dimensional space. The MgFE domain of the first type is obtained by rotating the generating FE around a specified axis at a specified (small) angle, and the MgFE domain of the second type is obtained by the parallel displacement of the generating FE along a specified straight line at a given distance. The nodes of the generating FE are the nodes of a coarse grid of the MgFE, and the nodes of any cross section of the coarse grid of the MgFE are the nodes of the generating FE. This approach simplifies the construction of approximating displacement functions on the coarse grids of the complex-shaped $\mathrm{MgFE}$, where the basis functions of the generating FE are used, and Lagrange polynomials are applied along the direction of the generating FE. The MgFE of the first type are used to calculate the composite shells of revolution, and the $\mathrm{MgFE}$ of the second type are used to calculate the composite cylindrical shells of revolution (with a variable curvature radius) and complex-shaped plates and beams. It is assumed that there are ideal bonds between the components of the inhomogeneous structure of the MgFE. The calculation of composite shells of double curvature using the MgFE of the first type is described in [16], and the application of the MgFE of the first and second types for determining the power elements of standard designs is demonstrated in [18].

\section{Multigrid FE of the first type. Complex-shaped composite shells of revolution}

The fundamental principles of constructing the MgFE of the first type, applied to analyze a three-dimensional SSS of composite shells of revolution, are considered on the example of a complex-shaped shell two-grid FE (2gFE), Fig. 1. For the $V_{d} 2 \mathrm{gFE}$, the following local coordinate systems are introduced: Cartesian $O x y z$, curvilinear $O \xi \eta \zeta$, and integer ijk for the nodes of the coarse grid $H_{d}$ of the $2 \mathrm{gFE}$. The nodes of the $H_{d}$ grid for the $2 \mathrm{gFE}$ are 36 in number and marked by dots in Fig. 1, with $c d$ denoting the shell axis. The generating singlegrid FE (1gFE) $V_{d}^{a}$ for the $V_{d} 2 \mathrm{gFE}$ has 12 nodes of the coarse grid $H_{d}$, denoted by dots in Fig. 2. The lateral sides of the $V_{d}^{a} 1 \mathrm{gFE}$ are parallel and extended by a dotted line to an the 
intersection with the $c d$ axis. The $V_{d}$ 2gFE domain is obtained by rotating the generating complex-shaped $1 \mathrm{gFE} V_{d}^{a}$ around the $c d$ axis at the specified angle $\alpha_{0}$ (corresponding to partitioning the shell into $2 \mathrm{gFE}$ ), and $\alpha_{0}$ is the apex angle of the $V_{d} 2 \mathrm{gFE}$. The basic partition $R_{d}$ of the $V_{d} 2 \mathrm{gFE}$ comprises three homogeneous $V_{e} 1 \mathrm{gFE}$ of the first order (described in detail in [12]), $e=1, \ldots, M$, and $M$ is the overall number of the $V_{e}$ FE. The partition $R_{d}$ accounts for the inhomogeneous structure and shape of the $2 \mathrm{gFE}$ and forms a fine grid $h_{d}$, where $H_{d} \subset h_{d}$. It is noteworthy that some nodes of the coarse grids of the MgFE can generally fail to match the nodes of the fine grids. We construct the $V_{e} 1 \mathrm{gFE}$ by using the equations of the three-dimensional problem of the elasticity theory [10], written in the local Cartesian coordinate system of the $V_{e}$ FE [12]. Thus, a three-dimensional SSS is implemented in the $2 \mathrm{gFE}$. Radii $R_{1}$ and $R_{3}\left(R_{2}\right.$ and $\left.R_{4}\right)$ describe the bottom (top) boundaries of the lateral edges of the $2 \mathrm{gFE}$. In the $H_{d}$ grid, the displacements functions $u_{d}, v_{d}$, and $w_{d}$, used to reduce the dimension of the basic partition $R_{d}$, are calculated.

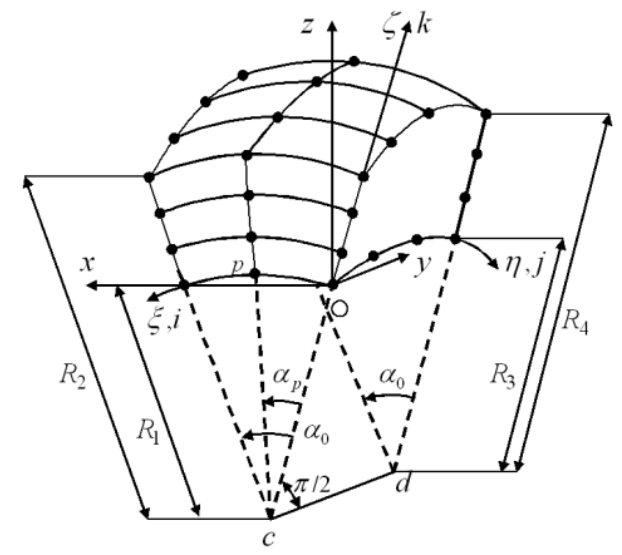

Fig. 1. Shell $2 \mathrm{gFE} V_{d}$.

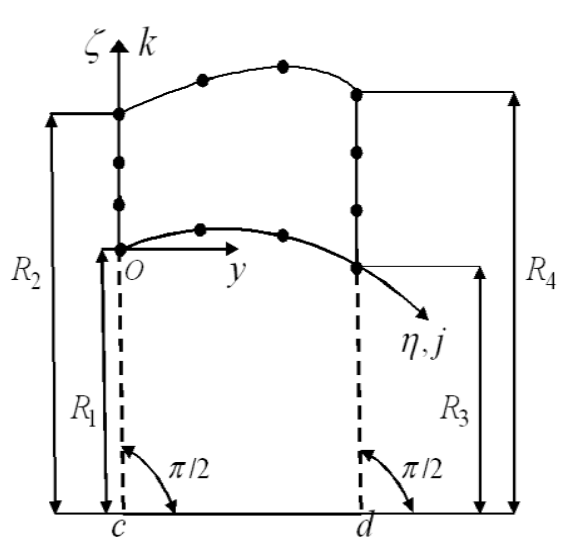

Fig. 2. Generating $1 \mathrm{gFE} V_{d}^{a}$.

The basis function $\psi_{i j k}$ for the $i, j, k$ node of the coarse grid $H_{d}$ of the $V_{d} 2 \mathrm{gFE}$ is sought in the form

$$
\psi_{i j k}(\alpha, y, \zeta)=N_{j k}(y, \zeta) L_{i}(\alpha),
$$

where $N_{j k}(y, \zeta)$ denotes the basis functions of the $j, k$ node of the $V_{d}^{a} 1 \mathrm{gFE}$, corresponding to the polynomial $P_{d}(y, \zeta)$ of the form [2]

$P_{d}=a_{1}+a_{2} y+a_{3} \zeta+a_{4} y \zeta+a_{5} y^{2}+a_{6} \zeta^{2}+a_{7} y^{2} \zeta+a_{8} y \zeta^{2}+$ $a_{9} y \zeta^{3}+a_{10} y^{3} \zeta+a_{11} y^{3}+a_{12} \zeta^{3}, \quad j, k=1, \ldots, 4, \quad L_{i}(\alpha)$ is the second-order Lagrange polynomial, $L_{i}(\alpha)=\prod_{p=1, p \neq i}^{3} \frac{\alpha-\alpha_{p}}{\alpha_{i}-\alpha_{p}}$, where $\alpha_{p}\left(\alpha_{i}\right)$ is the apex angle of the $p$ node ( $i$ node), $i=1, \ldots, 3$, Fig. 1 , and $\alpha$ is the apex angle of the $M$ point lying in the $V_{d} 2 \mathrm{gFE}$ domain.

Thus, the basis functions $\psi_{i j k}$ of the $V_{d} 2 \mathrm{gFE}$ of the first type are represented by power polynomials, which are the shape functions of the generating $\mathrm{FE} V_{d}^{a}$, and by the Lagrange polynomials in the direction of rotation of the generating FE around the specified axis. 
Denotations $N_{\beta}=\psi_{i j k}$, where $i=1, \ldots, 3 ; j, k=1, \ldots, 4 ; \beta=1, \ldots, 36$. Then, using (1), we write the displacement functions $u_{d}, v_{d}$, and $w_{d}$ as

$$
u_{d}=\sum_{\beta=1}^{36} N_{\beta} u_{\beta}, \quad v_{d}=\sum_{\beta=1}^{36} N_{\beta} v_{\beta}, \quad w_{d}=\sum_{\beta=1}^{36} N_{\beta} w_{\beta},
$$

where $N_{\beta}, u_{\beta}, v_{\beta}$, and $w_{\beta}$ denote the basis function and displacements of the $\beta$-th node of the $H_{d}$ grid.

The functional of the total potential energy $\Pi_{d}$ of the base partition $R_{d}$ of the $2 \mathrm{gFE}$ is written in the form

$$
\Pi_{d}=\sum_{e=1}^{M}\left(\frac{1}{2} \boldsymbol{\delta}_{e}^{T}\left[K_{e}\right] \boldsymbol{\delta}_{e}-\boldsymbol{\delta}_{e}^{T} \mathbf{P}_{e}\right)
$$

where $\left[K_{e}\right], \mathbf{P}_{e}$, and $\boldsymbol{\delta}_{e}$ denote the stiffness matrix, nodal force vectors, and displacement vectors of the $V_{e} 1 \mathrm{gFE}$, corresponding to the coordinate system $O x y z$ of the $V_{d} 2 \mathrm{gFE}$.

Equations (2) are used to express the nodal displacement vector $\boldsymbol{\delta}_{e}$ of the base $1 \mathrm{gFE}$ $V_{e}$ through the nodal displacement vector $\boldsymbol{\delta}_{d}$ of the coarse grid $H_{d}$ $\left(\boldsymbol{\delta}_{d}=\left\{u_{\beta}, v_{\beta}, w_{\beta}\right\}^{T}\right)$, i.e.

$$
\boldsymbol{\delta}_{e}=\left[A_{e}^{d}\right] \boldsymbol{\delta}_{d},
$$

where $\left[A_{e}^{d}\right]$ denotes the rectangular matrix, $e=1, \ldots, M$.

Equation (4) is substituted into Eq. (3) and the condition $\partial \Pi_{d}\left(\boldsymbol{\delta}_{d}\right) / \partial \boldsymbol{\delta}_{d}=0$ yields the relationship $\left[K_{d}\right] \boldsymbol{\delta}_{d}=\mathbf{F}_{d}$, where

$$
\left[K_{d}\right]=\sum_{e=1}^{M}\left[A_{e}^{d}\right]^{T}\left[K_{e}\right]\left[A_{e}^{d}\right], \quad \mathbf{F}_{d}=\sum_{e=1}^{M}\left[A_{e}^{d}\right]^{T} \mathbf{P}_{e} .
$$

Here $\left[K_{d}\right]$ is the stiffness matrix, and $\mathbf{F}_{d}$ is the nodal force vector of the $2 \mathrm{gFE} V_{d}$.

Let the vector $\boldsymbol{\delta}_{d}$ be determined. Equation (4) is used to calculate vector $\boldsymbol{\delta}_{e}$ of the nodal displacements of the $V_{e} 1 \mathrm{gFE}$ in the coordinate system $O x y z$ of the $V_{d} 2 \mathrm{gFE}$. The displacement vector $\boldsymbol{\delta}_{e}^{*}$ of the $V_{e} 1 \mathrm{gFE}$ (corresponding to the local Cartesian coordinate system of the $\left.V_{e} 1 \mathrm{gFE}\right)$ is sought for by the expression $\boldsymbol{\delta}_{e}^{*}=\left[M_{e}\right] \boldsymbol{\delta}_{e}$, where $\left[M_{e}\right]$ is the rotation matrix [2]. The vector $\boldsymbol{\delta}_{e}^{*}$ and the known algorithms of the FEM [1,2] are used to calculate the equivalent stress at the center of the $V_{e} 1 \mathrm{gFE}$ domain.

The calculations of the elastic composite round cylindrical shells and panels, carried out using MgFE, are described in [12]. The base functions of the coarse grids of the $2 \mathrm{gFE}$ of these shells are determined in the form of Lagrange polynomials and using the power polynomials $P(x, y, z)$ of the first, second, and third orders [12], written in the local Cartesian coordinate systems. The MgFE of the shells of revolution are verified using the known numerical method $[2,12]$.

Note 1. In view of Eq. (4), the dimension of the vector $\boldsymbol{\delta}_{d}$ (i.e., the dimension of $V_{d}$ $2 \mathrm{gFE}$ ) is independent of $M$, which is the total number of the base FE $V_{e}$ that comprise the 
$V_{d} 2 \mathrm{gFE}$ domain. Consequently, it is possible to use arbitrarily small basic partitions $R_{d}$, allowing one to arbitrarily exactly account for the complex shape and inhomogeneous (microinhomogeneous) structure of the $2 \mathrm{gFE} V_{d}$ and its complex fixing and loading, as well as to arbitrarily exactly describe a three-dimensional SSS in the $V_{d} 2 \mathrm{gFE}$ domain (with no increase in the dimension of the $V_{d} 2 \mathrm{gFE}$, which is noteworthy).

As shown by the calculations, as the basic partitions of the MgFE become finer, the solution errors decrease. Similarly, the procedures described in Sec. 2 are used to design the composite $2 \mathrm{gFE}$ for calculating complex-shaped composite rings and shafts with central circular holes. Three-grid FE ( $3 \mathrm{gFE})$ of the first type are designed using the $2 \mathrm{gFE}$ of the first type with the help of procedures similar to those in Sec. 2

You are free to use colour illustrations for the online version of the proceedings but any print version will be printed in black and white unless special arrangements have been made with the conference organiser. Please check whether or not this is the case. If the print version will be black and white only, you should check your figure captions carefully and remove any reference to colour in the illustration and text. In addition, some colour figures will degrade or suffer loss of information when converted to black and white, and this should be taken into account when preparing them.

\section{Multigrid FE of the second type}

\subsection{Multigrid FE for calculating complex-shaped composite beams}

The main provisions of the construction procedure for the MgFE of the second type used to analyze the three-dimensional SSS of composite beams are considered on the example of the $V_{p} 2 \mathrm{gFE}$ (Fig. 3), which is a complex-shaped rectilinear composite beam with a hole, with the hole section hatched in the figure. The domain of the $V_{p} 2 \mathrm{gFE}$ is obtained by the parallel displacement of the generating complex-shaped $1 \mathrm{gFE} V_{p}^{a}$ (Fig. 4) with a hole (hatched) along the $O y$ axis at the specific distance $d$. The basic partition $R_{p}$ of the $V_{p}$ $2 \mathrm{gFE}$ consists of the homogeneous $1 \mathrm{gFE} V_{e}$ of the first order, $e=1, \ldots, M$. The partition $R_{p}$ accounts for the inhomogeneous structure and complex shape of the $V_{p} 2 \mathrm{gFE}$ and forms a fine grid $h_{p}$, in which the coarse grid $H_{p}$ of the $2 \mathrm{gFE}$ is nested, and the nodes of the $H_{p}$ grid are marked by points (48 nodes, Fig. 3). The stress state in the $V_{e} 1 \mathrm{gFE}$ is described by the equations of the three-dimensional problem of the elasticity theory [10], written in the local Cartesian coordinate system of the $V_{e}$ FE. Consequently, a threedimensional SSS is implemented in the $V_{p}$ 2gFE domain. The nodes of the $V_{p}^{a} 1 \mathrm{gFE}$ of the third order are the nodes of the coarse grid $H_{p}$, marked by points (12 nodes). For the nodes of the $H_{p}$ grid, a coordinate system $i j k$ is introduced, in which $i, j, k=1, \ldots, 4$. 

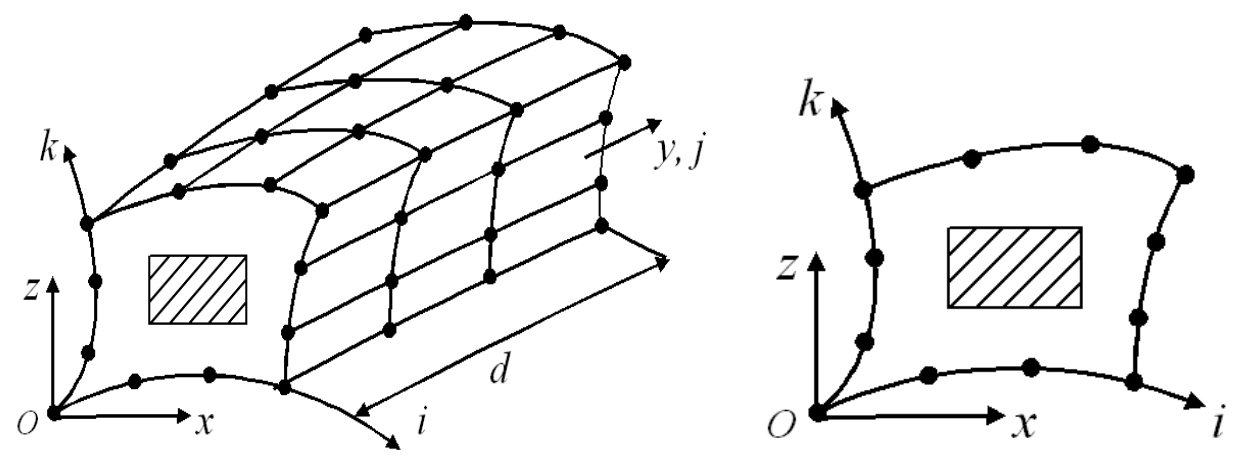

Fig. 3. Beam $2 \mathrm{gFE} V_{p}$.

Fig. 4. Generating $1 \mathrm{gFE} V_{p}^{a}$.

The basis function $\psi_{i j k}$ for the $i, j, k$ node of the coarse grid $H_{p}$ is determined as

$$
\psi_{i j k}(x, y, z)=N_{i k}(x, z) L_{j}(y),
$$

where $N_{i k}$ denotes the basis function of the $i, k$ node of the generating $\operatorname{FE} V_{p}^{a}$, corresponding to a polynomial of the form [2]

$$
\begin{gathered}
P(x, z)=a_{1}+a_{2} x+a_{3} z+a_{4} x z+a_{5} x^{2}+a_{6} z^{2}+a_{7} x^{2} z+a_{8} x z^{2}+ \\
a_{9} x z^{3}+a_{10} x^{3} z+a_{11} x^{3}+a_{12} z^{3},
\end{gathered}
$$

$i, k=1, \ldots, 4, L_{j}(y)$ is the Lagrange polynomial of the third order, having the form

$$
L_{j}(y)=\prod_{p=1, p \neq j}^{3} \frac{y-y_{p}}{y_{j}-y_{p}}
$$

where $j=1, \ldots, 4, y_{p}$ is the coordinate of the node $p$ of the coarse grid $H_{p}$, lying on the $O y$ axis (Fig. 3). Using Eq. (6), we write the displacement functions $u_{p}, v_{p}$, and $w_{p}$ for the $H_{p}$ grid in the form

$$
u_{p}=\sum_{\beta=1}^{48} N_{\beta} u_{\beta}, \quad v_{p}=\sum_{\beta=1}^{48} N_{\beta} v_{\beta}, \quad w_{p}=\sum_{\beta=1}^{48} N_{\beta} w_{\beta},
$$

where $u_{p}, v_{p}$, and $w_{p}$ denote the displacements of the $\beta$-th node of the coarse grid $H_{p}$, where $\beta=1, \ldots, 48$.

It is noteworthy that the displacement functions $u_{p}, v_{p}$, and $w_{p}$ are only used to reduce the dimension of the basic partition $R_{p}$ of the $V_{p} 2 \mathrm{gFE}$. The displacement functions (9) and algorithms similar to those in Sec. 2 are used to determine the stiffness matrix and the nodal force vector of the $V_{p} 2 \mathrm{gFE}$ of the second type.

Note 2. The proposed method allows designing $2 \mathrm{gFE}$ whose one characteristic dimension is significantly larger or smaller than all the others. In the $O y$ direction of the large dimension of the $V_{p}$ 2gFE, it is reasonable to use the high order of displacement approximation (i.e., the high order of the Lagrange polynomials $L_{j}(y)$ ), which allows constructing solutions with a small error 


\subsection{Multigrid FE for calculating complex-shaped composite cylindrical shells}

The construction procedure of the $2 \mathrm{gFE}$ of the second type, used to analyze the threedimensional SSS of cylindrical shells of an inhomogeneous structure and complex shape, is considered on the example of the $V_{e}^{a} 2 \mathrm{gFE}$ with characteristic dimensions of $17 h \times 24 h \times 18 h$ (Fig. 5), which has a hole and the section of which is hatched in the figure. The generatrix (straight line) of the median surface of the $V_{e}^{a} 2 \mathrm{gFE}$ is parallel to the $O y$ axis (Fig. 5). The $V_{e}^{a} 2 \mathrm{gFE}$ domain is obtained by the parallel displacement of the generating complex-shaped 1 gFE $V_{a}$ (Fig. 6) (the hole section is hatched) along the $O y$ axis at a specified distance $d=24 h$. The basic partition $R_{a}$ of the $V_{e}^{a} 2 \mathrm{gFE}$ consists of cube-shaped homogeneous $1 \mathrm{gFE} V_{e}$ of the first order with side $h, e=1, \ldots, M$, where $M$ denotes the total number of the $V_{e}$ FE. The partition $R_{a}$ accounts for the inhomogeneous structure and complex shape of the $V_{e}^{a} 2 \mathrm{gFE}$ and forms a fine grid $h_{a}$, in which the coarse grid $H_{a}$ of the $2 \mathrm{gFE}$ is nested $\left(H_{a} \subset h_{a}\right.$ ). The nodes of the coarse grid $H_{a}$ are marked in Fig. 5 by points (60 nodes). The nodes of the $V_{a} 1 \mathrm{gFE}$ of the third order are the nodes of the coarse grid $H_{a}$, marked by points (12 nodes, Fig. 6). A stress state in the $V_{e} 1 \mathrm{gFE}$ is described by the equations of the three-dimensional problem of the elasticity theory [10] (written in the local Cartesian coordinate system of the $V_{e} \mathrm{FE}$ ). Consequently, the three-dimensional SSS is implemented in the $V_{e}^{a} 2 \mathrm{gFE}$ domain.

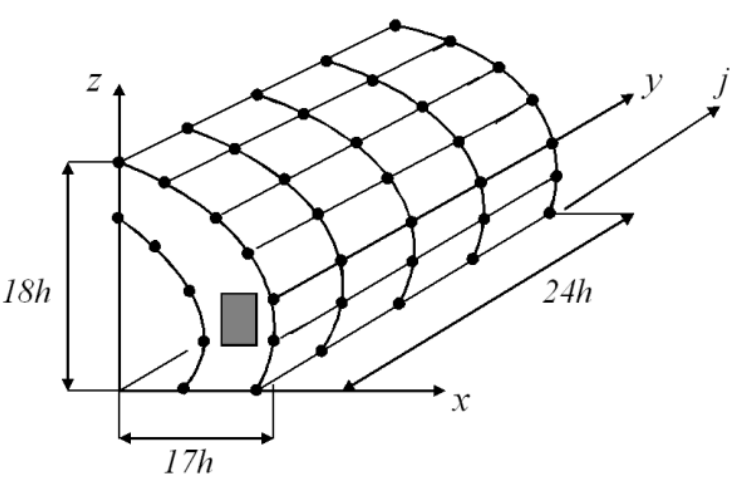

Fig. 5. $V_{e}^{a} 2 \mathrm{gFE}$.

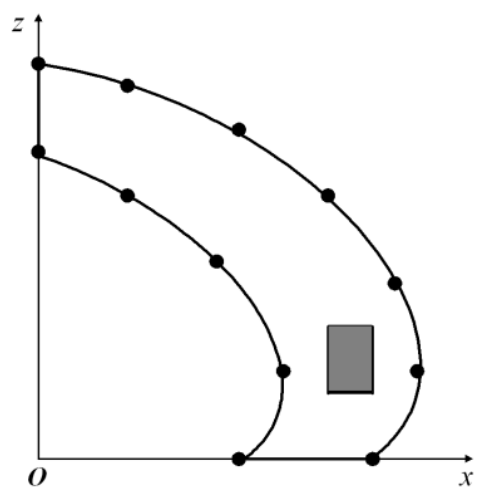

Fig. 6. Generating $1 \mathrm{gFE} V_{a}$.

For the pair of numbers $i, j$, where $i=1, \ldots, 12, j=1, \ldots, 5$, we determine an integer $\beta \geq 1, \beta=1, \ldots, 60$. The basis function $\psi_{\beta}(x, y, z)$ for the $\beta$-th node in the coarse grid of the $V_{e}^{a} 2 \mathrm{gFE}$ is sought in the form

$$
\psi_{\beta}(x, y, z)=N_{i}(x, z) L_{j}(y),
$$

where $\beta=\overline{1,60}, N_{i}(x, z)$ is the shape function of the $i$ th node of the generating $1 \mathrm{gFE} V_{a}$, $i=1, \ldots, 12$, corresponding to the polynomial $P(x, z)$, presented in the local Cartesian coordinate system $\mathrm{Oxz}$ (Fig. 6), of the form (7), $L_{j}(y)$ is the fourth-order Lagrange polynomial (Eq. (8)), where $j=1, \ldots .5, y_{p}$ is the coordinate of the $p$ node of the coarse grid $H_{a}$, lying on the $j$ axis, which is parallel to the $O y$ axis (Fig. 5). 
In Eq. (10), the basis functions $\psi_{\beta} 2 \mathrm{gFE} V_{e}^{a}$ of the second type are represented by power polynomials, i.e., functions of the form $N_{i}(x, z)$ of the generating FE $V_{a}$, and by the Lagrange polynomials $L_{j}(y)$ in the direction of the generating FE (along the $O y$ axis). Using Eq. (10), we write the displacement functions $u_{a}, v_{a}$, and $w_{a}$ for the coarse grid $H_{a}$ as

$$
u_{a}=\sum_{\beta=1}^{60} N_{\beta} u_{\beta}, \quad v_{a}=\sum_{\beta=1}^{60} N_{\beta} v_{\beta}, \quad w_{a}=\sum_{\beta=1}^{60} N_{\beta} w_{\beta},
$$

where $N_{\beta}, u_{\beta}, v_{\beta}$, and $w_{\beta}$ denote the basis function and displacements of the $\beta$-th node of the $H_{a}$ grid.

It is noteworthy that the displacement functions $u_{a}, v_{a}$, and $w_{a}$ are only used to reduce the dimension of the basic partition $R_{a}$ of the $2 \mathrm{gFE} V_{e}^{a}$. We use the displacement functions (11) and the algorithms similar to those in Sec. 2 to determine the stiffness matrix and the nodal force vector of the $V_{e}^{a} 2 \mathrm{gFE}$ of the second type.

\subsection{Multigrid FE for calculating complex-shaped composite plates}

We consider the construction procedure for the $2 \mathrm{gFE}$ of the second type in order to analyze the three-dimensional SSS of plates with an inhomogeneous structure on the example of the complex-shaped composite laminated $2 \mathrm{gFE} V_{g}^{b}$ (Fig. 7), where Oxyz is the Cartesian coordinate system. The characteristic dimensions $B$ and $H$ of the $V_{g}^{b} 2 \mathrm{gFE}$ significantly exceed the dimension of $h$, with $h$ being the thickness of the $2 \mathrm{gFE}$. The $V_{g}^{b} 2 \mathrm{gFE}$ domain is obtained by the parallel displacement of the generating $1 \mathrm{gFE} V_{g}$ (Fig. 8) along the $O y$ axis at the given distance $h$. The coarse grid of the $V_{g}^{b} 2 \mathrm{gFE}$ has 27 nodes marked by points in Fig. 7. The basic partition of the $V_{g}^{b} 2 \mathrm{gFE}$ consists of cube-shaped homogeneous FE of the first order (rectangular parallelepiped [2]), in which a three-dimensional SSS is implemented. It is noteworthy that the basic partitions of the $2 \mathrm{gFE}$ can be arbitrarily small, i.e., can arbitrarily exactly account for the inhomogeneous structure and complex shape of the $2 \mathrm{gFE}$. The base function $\psi_{\beta}(x, y, z)$ for the $\beta$-th node in the coarse grid of the $V_{g}^{b}$ $2 \mathrm{gFE}$ is sought in the form (6), where $\beta=1, \ldots, 27, N_{i}(x, z)$ is the shape function of the $i$-th node of the generating $1 \mathrm{gFE} V_{g}, i=1, \ldots, 9$, corresponding to a polynomial of the form $P_{g}(x, z)=a_{1}+a_{2} x+a_{3} z+a_{4} x z+a_{5} x^{2}+a_{6} z^{2}+a_{7} x^{2} z+a_{8} x z^{2}+a_{9} x^{2} z^{2}$, presented in the local Cartesian coordinate system $O x z$, Fig. 8, and $L_{j}(y)$ denotes the Lagrange polynomials of the second order. 


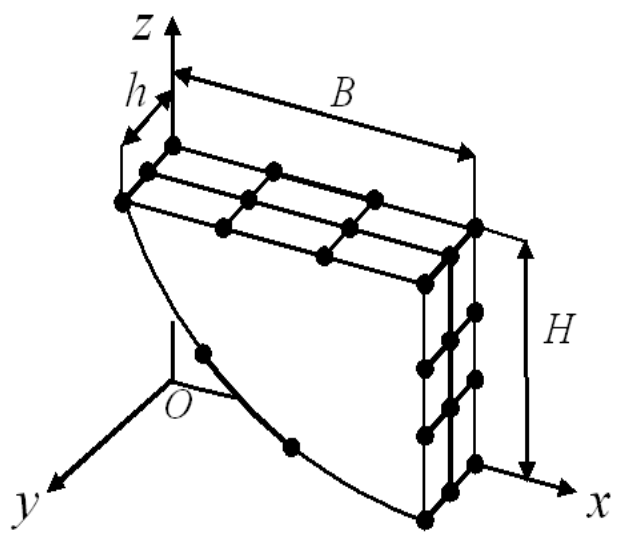

Fig. 7. Laminated $2 \mathrm{gFE} V_{g}^{b}$.

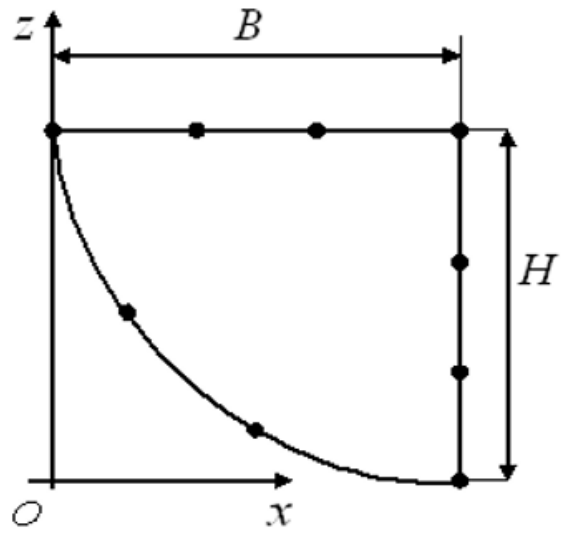

Fig. 8. Generating $1 \mathrm{gFE} V_{g}$.

The stiffness matrix and nodal force vector of the laminated $2 \mathrm{gFE} V_{g}^{b}$ are determined using procedures similar to those in Secs. 3.1 and 3.2. The discrete models of the plates are generally comprised of complex-shaped $2 \mathrm{gFE}$ (such as curvilinear $2 \mathrm{gFE} V_{g}^{b}$ ) and $2 \mathrm{gFE}$ shaped as a rectangular parallelepiped, constructed with the help of power polynomials [2], and Lagrange polynomials [12]. It is noteworthy that the proposed MgFE of the first and second types can be used in analyzing the three-dimensional SSS of corrugated plates, panels, and floors $[19,20]$ (corrugation can be shaped as a trapezium, rectangle, triangle, a part of a circular arc, etc.). Three-grid FE ( $3 \mathrm{gFE}$ ) of the second type are designed using the $2 \mathrm{gFE}$ of the second type with the help of procedures similar to those in Secs. 2, 3.2, and 3.3.

\subsection{Multigrid FE for calculating curvilinear composite beams}

We consider a curvilinear beam $l_{1}$ (frame beam). The $V_{L}^{a} 2 \mathrm{gFE}$ domain (Fig. 9), where the $2 \mathrm{gFE}$ approximates the beam $l_{1}$, is obtained by the parallel displacement of the generating $1 \mathrm{gFE} V_{L}$ (Fig. 10) along the $O y$ axis at the distance $d$, which is the beam width. The transverse section of the beam is $h \times d$, where $h$ is its height (thickness), corresponding to arc $d s$ (Fig. 9). The thickness of the transverse beam can be variable. The coarse grid of the $V_{L}^{a} \quad 2 \mathrm{gFE}$ has 24 nodes marked by points (Fig. 9).

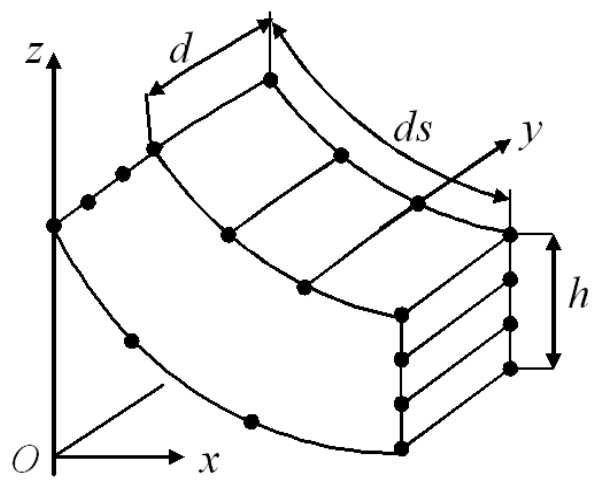

Fig. 9. Frame $2 \mathrm{gFE} V_{L}^{a}$.

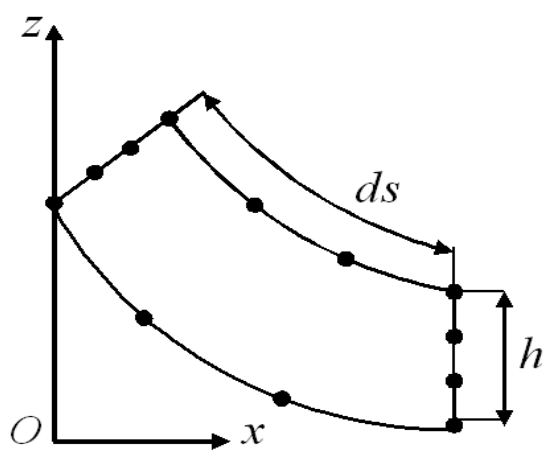

Fig. 10. Generating $1 \mathrm{gFE} V_{L}$. 
The basis function $\psi_{\beta}(x, y, z)$ for the $\beta$-th node of the coarse grid of the $V_{L}^{a} 2 \mathrm{gFE}$ is determined in the form (10), where $\beta=1, \ldots .24, N_{i}(x, z)$ denotes the shape function of the $i$-th node of the generating $1 \mathrm{gFE} V_{L}$ (Fig. 10), $i=1, \ldots, 12$, corresponding to a polynomial of the form (7), written in the local Cartesian coordinate system $O x z$ (Fig. 10), and $L_{j}(y)$ denotes the Lagrange polynomials of the first order. $i=1, \ldots, 12$. The stiffness matrix and nodal force vector of the $V_{L}^{a} 2 \mathrm{gFE}$ are determined in procedures similar to those in Secs. 3.2 and 3.3 .

\section{Conclusion}

In this paper, three-dimensional composite and homogeneous MgFE of two types of complex shapes are considered, which are designed with the use of forming FE. The procedures for the construction of type 1 and type $2 \mathrm{MgFE}$ are described, which are used for the calculation of composite (homogeneous) shells of rotation, cylindrical shells (with a variable radius of curvature), plates and beams of complex shape. The main advantages of the proposed $\mathrm{MgFE}$ are that they take into account the inhomogeneous, microinhomogeneous structure and complex shape of bodies, describe three-dimensional behavior in composite (homogeneous) bodies, form discrete models of small dimension and generate approximate solutions with a small error.

\section{References}

1. O.C. Zienkiewicz. The Finite Element Method in Engineering Science (McGraw-Hill, London, 1971)

2. D.H. Norrie and G. de Vries. An Introduction to Finite Element Analysis (Academic Press, New York, San Francisco, London, 1978)

3. A.P. Kiselev, N.A. Gureeva, R.Z. Kiselyova, Izv. Vuzov. Stroitel'stvo, 1, 106-112 (2010)

4. S.K. Golushko, Yu.V. Nemirovskii. Direct and Inverse Problems of the Mechanics of Elastic Composite Plates and Shells of Revolution (Fizmatlit, Moscow, 2008)

5. A. Ahmed A, S. Kapuria, Composite Structures, 158, 112-127 (2016)

6. E. Carrera, A. Pagani, S. Valvano, Composites Part B: Engineering, 111, $294-314$ (2017)

7. M.Y. Yasin, S. Kapuria, Composite Structures, 98, 202-214 (2013)

8. M. Cinefra, E. Carrera, Int. J. Num. Meth. Eng. 93, 2, 160-182 (2013)

9. M.F. Caliri, A. J.M. Ferreira, V. Tita, Composite Structures, 156, 63-77 (2016)

10. V.I. Samul'. Fundamentals of the Theory of Elasticity and Plasticity (Vysshaya Shkola, Moscow, 1982)

11. A.D. Matveev, Vestnik Krasnoyarskogo Gosudarstvennogo Agrarnogo Universiteta, 3, 44-47 (2014)

12. A.D. Matveev, A.N. Grishanov, Izvestia Altaiskogo Gosudarstvennogo Universiteta. Seriya: Matematika i Mekhanika, 4, 120-125 (2017) 
13. A. D. Matveev. The Multigrid Finite Element Method in Calculating ThreeDimensional Homogeneous and Composite Solids. Uchen. Zap. Kazan. Univ. Ser.: Fiz.-Matem. Nauki, 158, No. 4, 530-543 (2016)

14. A.D. Matveev, Vestnik KrasGAU, 11, 131-140 (2017)

15. A.D. Matveev, Vestnik KrasGAU, 2, 90-103 (2018)

16. A.D. Matveev, Vestnik KrasGAU, 3, 126-137 (2018)

17. A.D. Matveev, IOP Conf. Ser.: Mater. Sci. Eng. 158, 1, 012067, 1-9 (2016)

18. A.D. Matveev, Vestnik KrasGAU, 6, 141-154 (2018)

19. Y Xia, M.I. Friswell, E.I Saavedra Flores, Itern. J. Solids Structures 49, 14, 1453-1462 (2012)

20. N. Talbi, A. Batti, R. Ayad, Y.Q. Guo, Composite Structures 88, 280-289 (2009) 